\title{
University Health Network Achieves Over 85\% CPOE with Misys CPR, Improves Accuracy and Saves Time
}

The implementation of a computerized physician order entry (CPOE) and medication administration system may seem at first glance to be primarily an information technology (IT) challenge. Yet those institutions that have implemented these applications have discovered that success is often more dependent upon behavioural changes in the medical staff. The fact is, putting the world's most advanced technology at a physician's fingertips does not provide any guarantee that he or she will not simply bypass the system by scribbling out a prescription.

University Health Network (UHN) addressed this challenge head-on during its implementation of Misys CPR for CPOE and medical administration functionality. The UHN Project Management Team worked collaboratively with physicians and other members of the network's medical and administrative staff to ensure their inputs were considered in customizing the system and that key influencers understood the advantages of CPOE and supported the implementation effort.

The result is that, with the implementation process recently concluded, over $85 \%$ of the prescriptions ordered at UHN in the areas that have been implemented - essentially all of the inpatient areas - are already being processed through Misys CPR. "The success of our implementation has made it possible to put a large number of safety features into place that were impossible with the manual approach," said Matt Anderson, Chief Information Officer for UHN. "In addition, we have seen dramatic improvements in efficiency, such as a $28 \%$ reduction in the elapsed time from prescription order to administration."

"In the plastic surgery department, we customized the interface so that all of our common medications can be ordered with only a few clicks," added Dr. Peter Bray. "CPOE improves ordering accuracy since we don't have to worry about an inadvertent error or someone misreading or mishearing what we said."

\section{Expanding Misys CPR Use}

UHN began using the predecessor of Misys CPR in the late-1980s for a number of administrative applications such as registering and admitting patients. UHN's use of the software has increased greatly over the last several years, as it has successfully implemented CPOE for diagnostic testing and patient dietary management.

Leveraging the momentum generated by these wins, UHN then embarked on CPOE implementation - its most ambitious and potentially most rewarding effort ever. "This was probably the most important IT project in our history because it directly affects the safety and quality of care provided to our patients," 
said Stephanie Saull-McCaig, Director, Acute Information Management, Shared Information Management Services (SIMS). "The majority of adverse events or errors in any hospital are focused on the administration of drugs. We looked closely at the Misys CPR solution and determined that it did an excellent job of integrating order entry and the administration of medicines while making it possible to fully utilize the information contained within the system."

\section{Winning Staff Co- operation}

The biggest challenge in this project, as with other CPOE implementation efforts, was in obtaining the cooperation of the medical staff so that the system would actually be used. "We recognized from the very beginning this was much more than a simple IT implementation - it was an all-encompassing change management initiative," Anderson, the CIO, said. "Physicians are naturally quite attached to methods that they've been using for years, often since they began practising. Convincing them to devote the time and energy required to adopt new methods was not an easy process. It required a lot of time and effort to understand each department's requirements and provide the customization needed to ensure these requirements were not only met but exceeded."

The multifaceted project team put together at UHN - consisting of representatives from Information Te ch n ology, Nursing, Pharmacy and Physicians - made a special effort to gain the participation of staff members who are leaders and influencers, regardless of their official role. And rather than taking a "big bang" approach, the team focused on one department at a time. After spending time with each group to understand how their specific order process worked, the project team customized the workflow to meet the department's needs while at the same time helping them understand the capabilities of Misys CPR and providing user training.
Custom Version for Each Department, Each Patient The decision was made early on in the implementation process to standardize as much of the customization as possible across the entire hospital while recognizing that each department's varying needs required individual approaches.

For example, the Internal Medicine Department uses a team-based approach in which physicians, nurses and pharmacists in a unit all work together, meaning any one of them might be called upon to meet the needs of a particular patient.

Each surgeon, on the other hand, has a one-on-one relationship with his or her patients. The surgeons typically come in each morning to check on their patients and write orders, and then they are off to the operating room for the rest of the day. The Behavioural Medicine Unit, conversely, provides a more relaxed environment because of the absence of medical emergencies and the fact that many patients are treated in group sessions. The medications tend to be less complex in this area, and many patients receive them directly from a nurse who watches to be sure they are swallowed.

The EHR Project Team began by defining the workflow for each department and setting up screens to move the order through each step in the process. For example, the anesthetists operate in groups, each of whom, in turn, follows many different patients. The process of customizing the application for this department included defining the groups and providing each member of the group's staff with access to information on and the ability to write orders for each assigned patient.

The team-based approach of the Internal Medicine Department made it necessary to develop a unique alert system. In the past, when a physician wrote an order, he or she would lift up a mechanical flag next to the patient's chart to let the nursing staff know that there was a new order. Now, when a physician 
writes an order, a similar flag pops up on a chart that appears on the screen of all the nurses in the unit. The screen has the patients' names down the left side of the page and the different departments that might require action across the top, including medication, lab, diets, radiology, etc.

On the other hand, in each department's version of the Misys CPR system, individual patients have their own inbox that can be used to view lab results, radiology results and new medication orders. And each group has established shortcuts to create the most common orders, including drug name, frequency, dosage and route.

\section{Quick Adoption}

\section{Speeds Payback}

"This process achieved the

intended result of producing a CPOE system that meets the needs of each department and is easy to use," Saull-McCaig said. "While I have heard of many hospitals that have CPOE levels below $50 \%$, ours was above $85 \%$ the first time we measured it. As a result, we've improved quality and efficiency."

The most important benefit - patient safety - is also the most difficult to quantify. "The Misys CPR implementation has made available a

wide range of clinical support tools that did not exist in the past," Dr. Bray said. "As soon as the physician enters the order, but before it is accepted, Misys CPR searches its clinical database for drug-drug or drug-allergy interactions. The software generates an alert if any problem is identified. And thanks to the system's flexibility, we are continually adding alerts that detect a myriad of major and minor errors. For example, the system now alerts a doctor whenever he or she enters an order for anyone that is no longer an inpatient."

Safeguards in the software ensure that all of these orders are complete and leave no room for misinterpretation or data entry errors. For example, in a recent month, the system gener- ated over 5,000 clinical decision alerts. Each department has created its own standardized orders that cover the vast majority of its orders, which further reduces the potential for error. The exactness of electronic ordering has eliminated the potential for mistakes that were previously possible due to miscommunication, such as when doctors would stick their head out of their office and call down the hall to a nurse to place an order. CPOE also helps prevent the possibility that doctors could forget they have prescribed a certain drug and then repeat the prescription.

Once the order is entered, the speed at which information can be transmitted electronically makes for a substantial reduction in the time required for the order to be reviewed by the pharmacy and then administered. For example, the time from order entry to review by pharmacy has been reduced by $26 \%$. Doctors have also been able to save substantial time by cutting out visits to the three hospitals within UHN that took place only to put orders on paper charts. Now they can log in from their office and enter orders for all their patients electronically.

"The implementation of CPOE with Misys CPR set off an intensive change management process that has touched every department and level within our organization," Saull-McCaig concluded."The results have clearly justified this effort. It's

difficult to quantify the improvement in patient safety, because our previous methods were not capable of tracking errors, but it's clear that we have made major strides in this area. We have also seen significant time savings among the medical, pharmacological and administrative staffs." 